\title{
SELECTIVE EFFECTS OF SECRETAGOGUES ON INSULIN SECRETION: A MATHEMATICAL MODEL
}

\author{
David Marshall Cohen \\ Applied Dynamics International, 3800 Stone School Road, Ann Arbor, MI 48104, U.S.A.
}

and

Sumer Belbez PeK

Department of Internal Medicine (Division of Endocrinology and Metabolism and the Metabolism Research Unit), The University of Michigan, Ann Arbor, MI 48109, U.S.A.

(Received 15 July 1982; in revised form 7 June 1983)

\begin{abstract}
A mathematical model of the secretion of insulin from pancreatic islets of Langerhans is proposed. Previously proposed mathematical models of insulin secretion have dealt solely with glucose-stimulated release, and not with the more complex patterns of secretion (such as "offresponses") in response to other secretagogues or combinations of secretagogues.

We conclude that (1) an off-response is consistent with a compartmental model; and (2) the facilitating effect of a constant concentration of glucose on leucine-stimulated insulin secretion is consistent with a selective effect of glucose on a single compartment of the model
\end{abstract}

Mathematical Model Compartment Insulin Secretion Off-response

\section{INTRODUCTION}

Mathematical models which simulate observed complex phenomena are useful tools to explore the mechanisms which underlie these phenomena and to predict the changes in these phenomena when the system is perturbed.

The secretion of insulin from the pancreatic islets of Langerhans is a complex process. Nutrients, such as glucose and certain amino acids, are the principal stimulators (secretagogues) of insulin secretion (Hedeskov [1]). Other inputs, such as neural or hormonal influences, play important roles in the modulation of insulin release (Smith and Porte [2]). The signals which elicit insulin secretion are likely to be generated when the stimulating substance activates specific sites in the plasma membrane of the insulinproducing cell, or when the secretagogue enters the cell and is metabolized. Signals thus generated may promote secretion as well as synthesis of insulin. The secretion process is associated with major ionic fluxes and depolarization in the cell membrane. The secretory granules containing insulin move toward the cell membrane and align themselves along contractile elements (microtubules). Microtubules facilitate the fusion of the membrane of the secretory granule with the plasma membrane of the cell. This fusion permits the release of insulin molecules in to the blood stream (exocytosis). Energy-requring steps are involved both in the synthesis and in the secretion of insulin.

Extensive information is available on the patterns of secretion of insulin, based on measurements of immunoreactive insulin released from the pancreas into the vessels draining the organ. The release patterns vary considerably, depending upon the nature of the secretagogue or combinations of secretagogues, the availability of energy sources and ions, as well as neural and hormonal influences. Several phases of insulin release are recognized. The acute phase occurs within minutes (and sometimes seconds) after the secretagogue reaches the insulin-produced cell. Despite continued exposure to the secretagogue, the acute phase dissipates within a few minutes. The late phase of insulin secretion follows the acute phase and is sustained as long as the pancreatic islets are exposed to the secretagogue. Under special circumstances, upon the reduction in the concentration of a secretagogue, a third phase of 


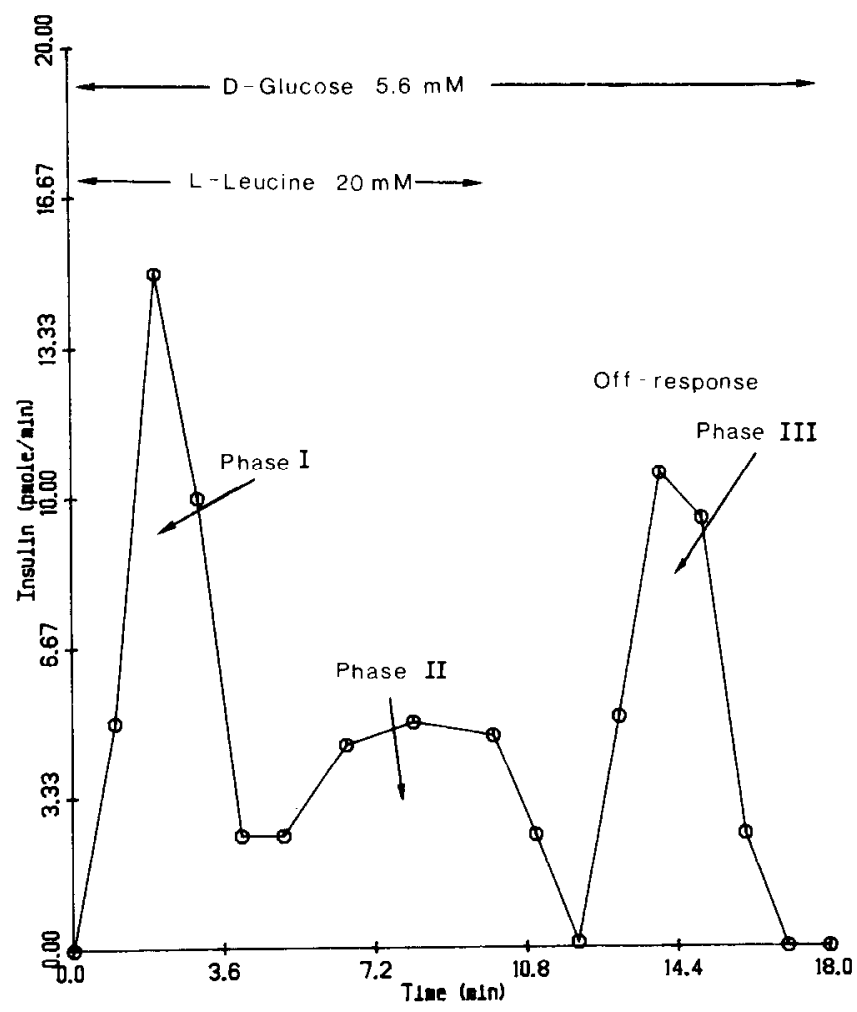

Fig. 1. Multiphasic secretion of insulin. Portal venous effluent levels of immunoreactive insulin observed in experiments using the isolated, perfused, rat pancreas. The values were converted from

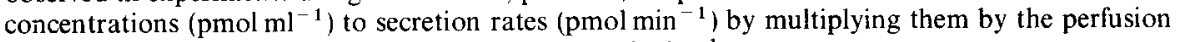
flow rate $\left(2.5 \mathrm{ml} \mathrm{min}^{-1}\right)$.

secretion is observed: the secretion rate first returns to basal levels, then increases rapidly several minutes later and finally dissipates within another few minutes. This third phase of insulin release is referred to as the "off-response" (Matschinsky et al. [3]). A schema of the three phases of insulin secretion is given in Fig. 1. Depending on the prevailing conditions, these phases may occur individually or in any combination, at varying magnitudes. The magnitude of the amount of hormone secreted during any of the phases may be influenced also by antecedent events. For example, antecedent administration of glucose will potentiate the release of insulin in response to a second administration of glucose (Grill et al. [4]).

The knowledge of the mechanisms of the multiphasic secretion of insulin is sketchy. Multiple hypotheses have been proposed but not adequately tested. Certain known influences appear to play preferential roles in the individual phases of secretion. For example, alpha-adrenergic neural modulation seems to dominate during the acute phase of secretion (Porte et al. [5]). We have proposed that the metabolism of glucose is mandatory for the insulin off-response which occurs in conjunction with the administration of leucine (Tai et al. [6]).

Several mathematical models for the stimulation of secretion of insulin by glucose have been proposed (Grodsky [7, 8], Bergman and Urquhart [9], Cerasi et al. [10], Cerasi [11], Licko and Silvers [12], Guyton et al. [13], Hagander et al. [14], Cobelli et al. [15], Toffolo et al. [16]). These models have dealt exclusively with the acute and late phases of glucoseinduced insulin rclcasc. Bergman and Bucolo [17] proposed a model of the stimulation of insulin secretion by a mixture of amino acids as well as by glucose. The ability of these models to deal with the complexities of the interactions of multiple secretagogues or with the offresponse has not been documented. The translation of the components proposed in these mathematical models into biological mechanisms may pose difficulties.

In this report, we propose a new mathematical model of insulin secretion. The model can 
accommodate all phases of insulin secretion induced by one or more secretagogues, as well as the influences of antecedent exposure to secretagogues. We incorporated into the model modules of mathematical compartments which influence one another. The rationale for the modular design was to facilitate the translation of the mathematical equations to biological events, as the knowledge of these events advanced. Conservatively, we opted for the inclusion of four mathematical compartments. One compartment is the locus of output from the system and represents the exit of insulin secretory granules from the islets. Three other compartments were included, each to represent a single metabolic event or group of intimately related metabolic events. By including four compartments in the model, we provided ourselves with sufficient flexibility to test the hypothesis that discrete individual or composite biological events have predominant effects of producing either an acute-phase response, a late-phase response, or an off-response. In designing and validating the model, we relied on actual data on the dynamics of insulin secretion generated in recent years in our laboratory during experiments with the isolated perfused rat pancreas.

Our modeling technique provides a mathematical reconstruction of the secretory curves. We extend the usual procedures for fitting data to curves to allow the inference of the particular changes to the system caused by the administration of certain secretagogues.

As shown in this report, our mathematical model successfully reproduces all patterns of insulin secretion observed in our biological model. In our mathematical model, the offresponse can be generated by modifying the input to a single compartment in a manner consistent with selective modification by glucose of the effect of leucine on that compartment.

\section{MATERIALS AND METHODS}

\section{Experimental methods}

The procedures for isolating and perfusing the rat pancreas, and for the measurement of perfusate levels of immunoreactive insulin have been reported previously (Pek et al. [18]). Under anesthesia, the large vessels conducting blood to and from the pancreas (the aorta and portal vein, respectively) were cannulated and the pancreas, together with its cannulated vessels, was removed surgically. The pancreas was placed in an extracorporeal perfusion apparatus. The artificial perfusion solution was Krebs-Ringer bicarbonate buffer which entered the pancreas through the aortic cannula and exited through the portal venous cannula. Each sample of pancreatic effluent which was collected from the portal vein represented the cumulative secretion during a $1 \mathrm{~min}$ time interval (at a flow rate of $2.5 \mathrm{ml} \mathrm{min}^{-1}$ ).

For the experiment using glucose alone, the basal concentration of D-glucose ("glucose") in the perfusate was $5.6 \mathrm{mM}$; it was increased twice to $16.7 \mathrm{mM}$, from minutes $5-35$ and from minutes 60-90. The switching of glucose solutions was executed within a fraction of a second, without interruption in the flow rate or pressure.

L-leucine ("leucine") was administered in three experiments. In two of these experiments, the concentration of glucose was held constant at $5.6 \mathrm{mM}$, while the concentration of leucine alternated between $0.0 \mathrm{mM}$ and $5.0 \mathrm{mM}$ or between $0.0 \mathrm{mM}$ and $20.0 \mathrm{mM}$. In the third experiment, glucose was absent, theophylline was present at a concentration of $10.0 \mathrm{mM}$, and the concentration of leucine alternated between $0.0 \mathrm{mM}$ and $5.0 \mathrm{mM}$. Theophylline is an amplifier of islet hormone secretion, most likely because it causes the accumulation of cyclic AMP. Theophylline magnifies the "on-response" to leucine, without altering the phasic pattern of leucine-induced insulin secretion (Landgraf-Leurs et al. [19]).

\section{Model assumptions and development of the mathematical model}

The focus of the modeling technique is on the stimulatory and inhibitory effects which secretagogues have on intraislet modulators of secretion. At the present time, the dynamic interrelationships among these modulators are not known (Hedeskov [1], Smith and Porte [2]). We are constructing a model of an incompletely specified system, in an attempt to gain understanding and insight into the underlying secretory mechanism.

Our mathematical model of hormone secretion rests on the notion that the pancreatic 


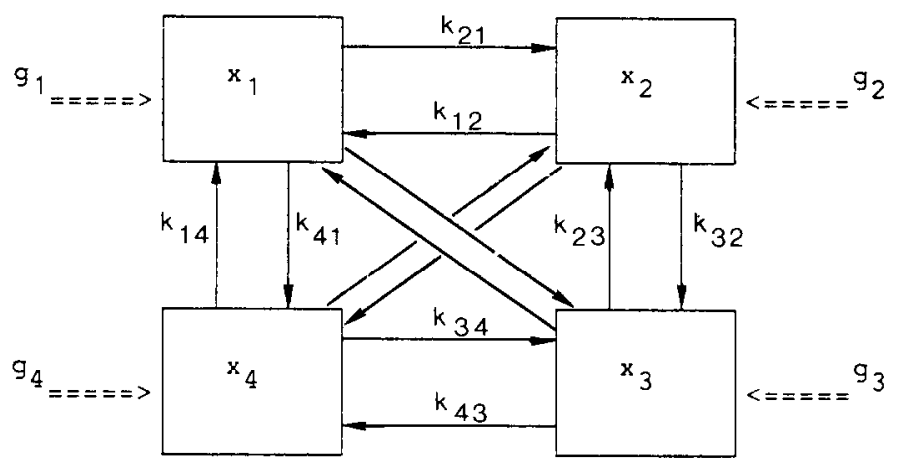

Fig. 2. Four-compartment mathematical model of insulin secretion. Each box corresponds to one compartment. Subscripts are used to indicate component numbers of vectors and coefficients. $\mathbf{g}$ $=$ input vector from the environment to the compartments; $k_{i j}=$ influence coefficient derived from the matrix $K$, representing the influence of compartment $j$ on compartment $i ; \mathbf{x}=$ state vector, representing the activity level in each compartment which results from the inputs $g$ and the influence coefficients $k_{i j}$. The activity level of compartment 4 corresponds to insulin secretion.

islets can be considered a multidimensional open system in which the rate of insulin secretion constitutes a component of the state (vector) variable. The changes in the insulin-secretory rate which occur in response to one or more secretagogues can be viewed as the transition curve from one steady state of secretion to a new steady state.

Our conceptual model consists of a set of interacting "compartments", each of which will be associated with certain biological energy transformations. We shall define the "activity level" of a compartment to be some scalar measure . the strength of the relevant biological events of that compartment.

The model has one state vector $(\mathbf{x})$, whose value determines the future behavior of the system in response to the environment. The equation of state is the following linear ordinary differential equation

$$
\dot{\mathbf{x}}=K \mathbf{x}+\mathbf{g}
$$

where boldface characters indicate vector quantities and $\operatorname{dot}(\cdot)$ indicates time derivative. $K$ is a real $4 \times 4$ (square) matrix and $\mathbf{x}$ and $\mathbf{g}$ are vectors, each having four components. This vector differential equation describes the behavior of the activity level of each compartment, and is merely a concise means of writing four differential equations (one for each compartment). The matrix $K$ is a matrix of "influence coefficients", an array of four rows and four columns which quantifies the influence of the activity level of one compartment on another. $K(i, j)$ represents the influence of the activity level of compartment $j$ on the activity level of compartment $i$. The vector $\mathbf{g}$ is the "input vector", representing the influence of the environment on our open system. Positive values of the entries of the matrix $K$ or of the vector g represent stimulatory influences, and negative values represent inhibitory influences.

For the purpose of obtaining an explicit solution for the state vector $\mathbf{x}$, we considered the case in which the concentrations of secretagogues were changed almost instantaneously (in comparison to the actual response time of the biological system under study). In addition, in order to simplify the construction of a particular instance of the model that will fit the experimental data, we assumed that $K$ is unaffected by the concentrations of secretagogues. This means that $K$ and $g$ were held constant, except for very short time periods ("critical time points") when $\mathbf{g}$ assumed new values. The relationships of $K, \mathbf{g}$ and $\mathbf{x}$ are given schematically in Fig. 2.

The activity level of compartment 4 corresponded to the rate of hormone secretion. The solution to the state equation was

$$
\mathbf{x}(t)=e^{K t}\left(\mathbf{x}(0)+K^{-1} \mathbf{g}\right)-K^{-1} \mathbf{g}
$$

and the activity level $x_{4}(t)$ of the fourth compartment could be written as a sum of 
exponentials and a steady state

$$
x_{4}(t)=a_{0}+a_{1} e^{\lambda_{1} t}+a_{2} e^{\lambda_{2} t}+a_{3} e^{\lambda_{3} t}+a_{4} e^{\lambda_{4} t} .
$$

Constraints on the components $\left(a_{i}, i=1,2,3,4\right)$ of the coefficient vector and on the eigenvalues $\left(\lambda_{i}, i=1,2,3,4\right)$ of the matrix $K$ were derived (Cohen [20]).

Since we hypothesized that secretagogues affected the vector $\mathbf{g}$ of inputs only, the concentrations of secretagogues could be identified with the input to the abstract (mathematical) system. The biological interpretation of the action of secretagogues in the mathematical model is that secretagogues increase or decrease the rates of biological energy transformations occurring within the hormone-producing cells.

Calculations were performed for the analysis of experimental data in three steps: (1) curve fitting; (2) selection of the parameters of the model; and (3) determination of the inputs to the model.

\section{Curve fitting}

First, curve approximations were constructed of experimental data in which the secretory rate was measured as a function of time. The equations of these curves (see above) consisted of the sum of a steady state value and four exponential terms. Using a well-known algorithm (Lanczos [21], Hamming [22]) we computed a single set of eigenvalues for all of the experimental data. The steady state value and the coefficients of the exponential terms were computed by a least squares method (Ravindran [23, 24], Proll [25], Cohen [20]).

\section{Selection of the parameters of the model}

Having computed the curve approximations for several experiments, we then selected the values of the intercompartmental influence coefficients of the mathematical model. The matrix $K$ of these coefficients is completely determined by its eigenvalues and the corresponding eigenvectors. The eigenvalues of $K$ are the same eigenvalues that were used in the curve approximations. The eigenvectors were chosen so that for the resulting matrix $K$, a simultaneous perturbation of pairs of compartments could result in either (1) an acute phase response; (2) a late phase response; or (3) an off-response.

In order to obtain adequate curve approximations, discontinuities with respect to time in the activity levels of the compartments were introduced; they occur at the times when the concentration of one or more secretagogues was changed. We decided not to allow discontinuities in the activity level of the compartment representing insulin secretion. The per cent change in the activity level of any compartment at the times of the discontinuities could be made as small as desired (Cohen [20]).

\section{Inferences about inputs to the model}

The selection of the parameters (intercompartmental influence cuefficients) of the model completed the specification of the model. The input vector $\mathbf{g}$ of the model for a particular combination of secretagogues was calculated from the coefficients of the corresponding curve approximation (Cohen [20]). The change $\Delta \mathbf{g}$ in the input vector that occurred when the concentration of a secretagogue was increased was computed by taking the (vector) difference of two values of the input vector $\mathbf{g}$. Upon comparing the vectors $\Delta \mathbf{g}$ for different secretagogues or combinations of secretagogues we deduced their selective (or preferential) effects on the compartments of the mathematical model.

\section{Computing facilities}

The programs were written in the FORTRAN IV programming language and executed on the University of Michigan's "MTS" timesharing system, using the Amdahl 470V/8 computer.

\section{RESULTS}

The steady state values and coefficient vectors of the curve approximations are tabulated in 
Table 1 . The steady state values and coefficient vectors for time segments $1,2,3$ and 4 , corresponding to the curve approximations of Figs $3,7,8,9,10,11$ and 12

\begin{tabular}{|c|c|c|c|c|c|c|}
\hline \multirow[b]{2}{*}{ Figure } & \multirow{2}{*}{$\begin{array}{c}\text { Time } \\
\text { segment }\end{array}$} & \multirow{2}{*}{$\begin{array}{c}\text { Steady-state } \\
\text { value }\end{array}$} & \multicolumn{4}{|c|}{ Coefficient vectors } \\
\hline & & & Component 1 & Component 2 & Component 3 & Component 4 \\
\hline \multirow[t]{4}{*}{3} & 1 & 27.403 & -28.786 & 7.159 & -9.456 & 3.692 \\
\hline & 2 & 0.010 & 0.065 & -0.924 & 8.635 & -4.428 \\
\hline & 3 & 27.403 & -23.886 & -19.389 & 70.207 & -54.269 \\
\hline & 4 & 0.010 & 0.056 & -1.030 & 17.954 & -9.539 \\
\hline \multirow[t]{4}{*}{7} & 1 & 0.000 & -0.003 & 0.373 & -0.486 & 0.185 \\
\hline & 2 & 0.000 & 0.036 & 1.185 & -2.123 & 0.902 \\
\hline & 3 & 0.000 & 0.000 & 0.497 & -0.709 & 0.255 \\
\hline & 4 & 0.000 & 0.051 & 1.275 & -2.303 & 0.981 \\
\hline \multirow[t]{4}{*}{8} & 1 & 24.718 & -25.098 & 45.674 & -77.927 & 32.706 \\
\hline & 2 & 0.010 & 0.704 & 16.192 & -37.343 & 21.872 \\
\hline & 3 & 24.718 & -24.007 & 87.871 & -151.922 & 63.947 \\
\hline & 4 & 0.010 & 3.751 & 16.460 & -42.138 & 24.697 \\
\hline \multirow[t]{2}{*}{9} & 1 & 0.000 & 0.236 & 6.489 & -11.594 & 4.921 \\
\hline & 2 & 0.000 & 0.088 & -0.306 & 0.929 & -0.440 \\
\hline \multirow[t]{2}{*}{10} & 1 & 5.929 & -6.373 & 9.687 & 4.454 & -13.593 \\
\hline & 2 & 0.138 & -0.104 & 0.056 & -0.171 & 0.081 \\
\hline \multirow[t]{2}{*}{11} & 1 & 3.898 & -2.952 & -3.231 & 4.360 & -2.058 \\
\hline & 2 & 2.693 & -2.827 & 1.002 & 1.788 & -1.377 \\
\hline \multirow[t]{2}{*}{12} & 1 & 14.037 & -14.220 & 2.069 & -3.211 & 1.343 \\
\hline & 2 & 0.000 & -0.017 & 5.161 & 42.530 & -46.249 \\
\hline
\end{tabular}

Table 2. The components of the discontinuity vector for the curve approximations in Figs 3, 7 and 8 . The discontinuity at the first and third critical time points is zero. Figure 9 is not included because the discontinuity vector is zero (only one dose of the sccretagogue was administered)

\begin{tabular}{cccccc}
\hline Figure & $\begin{array}{c}\text { Critical } \\
\text { time point }\end{array}$ & Component 1 & Component 2 & Component 3 & Component 4 \\
\hline 3 & 2 & 388.788 & -737.113 & 21.764 & 0.000 \\
& 4 & 476.175 & -677.709 & 20.124 & 0.000 \\
7 & 2 & -23.106 & 41.880 & -1.258 & 0.000 \\
& 4 & -24.596 & 43.952 & -1.321 & 0.000 \\
& 2 & -1459.605 & 2682.007 & -80.444 & 0.000 \\
& 4 & -1502.177 & 2652.100 & -79.613 & 0.000 \\
\hline
\end{tabular}

Table 3. The matrix $N$ of cigenvectors and the matrix $K$ of influcnec cocficients corresponding to the list of eigenvalues given in the text. $K(i, j)$ is the strength of the influence of compartment $j$ on compartment $i$

$\begin{array}{rr}-13.231 & -7.407 \\ -30.731 & 12.20 \\ 0.880 & -0.37 \\ 1.000 & 1.000 \\ & \\ -0.294 & -3.52 \\ 0.552 & 7.03 \\ -0.013 & -0.28 \\ 0.160 & -3.88\end{array}$

N

K 
Table 4. The components of the change $\Delta \mathbf{g}$ in the input vector for the curve approximations in Figs $3,7,8,9,10,11$ and 12. The value of $\Delta \mathrm{g}$ for the curve approximations in Figs 4,5 and 6 is the same as for Fig. 3

\begin{tabular}{rrrrr}
\hline \multicolumn{1}{c}{ Figure } & \multicolumn{1}{c}{ Component number } & \multicolumn{1}{c}{4} \\
\hline 3 & -212.970 & 2 & \multicolumn{1}{c}{3} & \multicolumn{1}{c}{46.684} \\
7 & 4.626 & 384.796 & -10.492 & 0.061 \\
8 & 883.542 & -8.688 & 0.258 & 0.004 \\
9 & 4.525 & -1669.454 & 49.167 & -0.025 \\
10 & -1.000 & -8.565 & 0.250 & 0.000 \\
11 & 0.000 & 0.000 & 0.000 & -0.442 \\
12 & 0.000 & -1.000 & 0.000 & -50.611 \\
\hline
\end{tabular}

Table 5. Root-mean-square errors of curve approximations presented in Figs 3, 5, 7,8,9,10,11 and 12

\begin{tabular}{cc}
\hline Figure & Error (in pmol min ${ }^{-1}$ ) \\
\hline 3 & 0.861 \\
5 & 0.927 \\
7 & 0.105 \\
8 & 3.032 \\
9 & 0.171 \\
10 & 0.557 \\
11 & 0.129 \\
12 & 0.691 \\
\hline
\end{tabular}

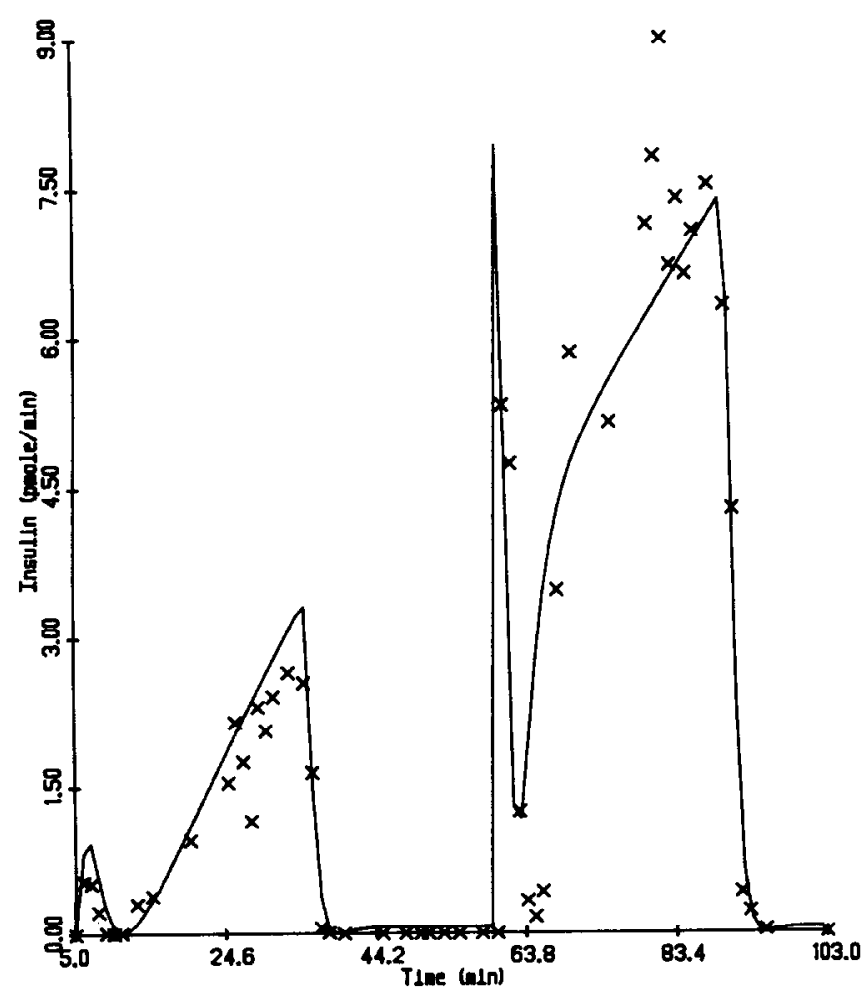

Fig. 3. Raw data $(X)$ and curve approximation (solid line) for glucose-stimulated insulin secretion (experiment P-1262-I). Two successive stimuli of glucose were administered, (between minutes 5-35 and 60-90) and two biphasic patterns were observed. The interval between the stimuli was $25 \mathrm{~min}$. The concentration of glucose was changed from $5.6 \mathrm{mM}$ to $16.7 \mathrm{mM}$ during the periods of stimulation. 
Table 1 and the discontinuity vectors are given in Table 2 . These tables contain sufficient information to reproduce each of the curve approximations. Technical details may be found in Cohen [20]. The order of the eigenvalues was taken to be the following: $-0.006,-0.489$, -1.25 and -2.3 .

The entries of the matrices $N$ and $K$, which specify the interrelationships of the compartments, are presented in Table 3 , and the derived components of the change in the input vector are given in Table 4. The latter table summarizes the direct effect of the secretagogues on the compartments of the model.

The square root of the average of the squares of the differences between the predicted and the measured rates of insulin secretion provides a quantitative evaluation of the curve approximations. These "root-mean-square errors" are given in Table 5. The larger the error, the poorer is the approximation.

\section{Biphasic pattern and long-term potentiation}

The biphasic pattern of insulin secretion, such as that seen in response to a rapid rise in the concentration of glucose, is twice illustrated in Fig. 3 : from minutes 5 to 35 and from minutes 60 to 90 . In both cases, the biphasic pattern followed the increase in the concentration of glucose from $5.6 \mathrm{mM}$ to $16.7 \mathrm{mM}$. The acute phase of secretion following the first stimulus occurred within minutes 5-9, and following the second stimulus, during minutes 60-63. The end of Phase 1 was demarcated by a dip (relative minimum) in the secretory rate. Following the acute phase occurred the "late" phase (minutes 9--35 and 63-90) in which the secretory rate increased, although not as precipitously as during the acute phase.

The potentiated biphasic response to the second glucose stimulus (minutes 60-90) relative to the first stimulus (minutes 5-35) was evident, both in the raw data and in the curve approximation.

In Fig. 3 the interval between successive glucose stimuli (step function increases from $5.6 \mathrm{mM}$ to $16.7 \mathrm{mM}$ ) was $25 \mathrm{~min}$. The model's predictions of the secretory rate for in tervals of 10, 15 and 55 min between stimuli are given in Figs 4, 5 and 6, respectively. In each case, the biphasic response occurred twice (once for each increase in the concentration of glucose) and with greater magnitude during the second stimulus. Experimental data from our laboratory corresponding to the 15 -min interval are plotted along with the curve approximation (Fig. 5).

We compared the potentiation of insulin secretion for two of the abovementioned predictions of the model. For the 10-min time interval (Fig. 4) the amount of insulin secreted in response to the second stimulus was $394 \%$ of the amount secreted in response to the first stimulus. When the time interval was $55 \mathrm{~min}$ (Fig. 6), the change was $393 \%$. Thus the long-term potentiating effect of the first administration of glucose on the amount of insulin released in response to the second stimulus remained the same even though the time period between the stimuli was increased from 10 to $55 \mathrm{~min}$.

\section{Triphasic patterns and long-term potentiation}

Two triphasic patterns of insulin secretion are demonstrated in Fig. 7. Glucose was present in the perfusion medium at a concentration of $5.6 \mathrm{mM}$ throughout the experiment. The concentration of leucine was raised from $0.0 \mathrm{mM}$ to $5.0 \mathrm{mM}$ at minute 5 and lowered to $0.0 \mathrm{mM}$ again at minute 15 . A phasic secretory pattern was observed during the $10 \mathrm{~min}$ of administration of leucine. Following the removal of leucine, a third phase ("off-response") began, and lasted until minute 25 , when the concentration of leucine was again increased to $5.0 \mathrm{mM}$. A potentiated acute phase was then observed, which was followed by a small second phase. The administration of leucine was stopped at minute 35 ; shortly thereafter, a potentiated off-response (relative to the earlier one) was observed, which lasted about $8 \mathrm{~min}$.

\section{Effect of different concentrations of the same secretagogue}

When the concentration of leucine alternated between $0.0 \mathrm{mM}$ and $20.0 \mathrm{mM}$, the pattern of insulin secretion (Fig. 8) was similar in shape, but magnified more than forty times when 


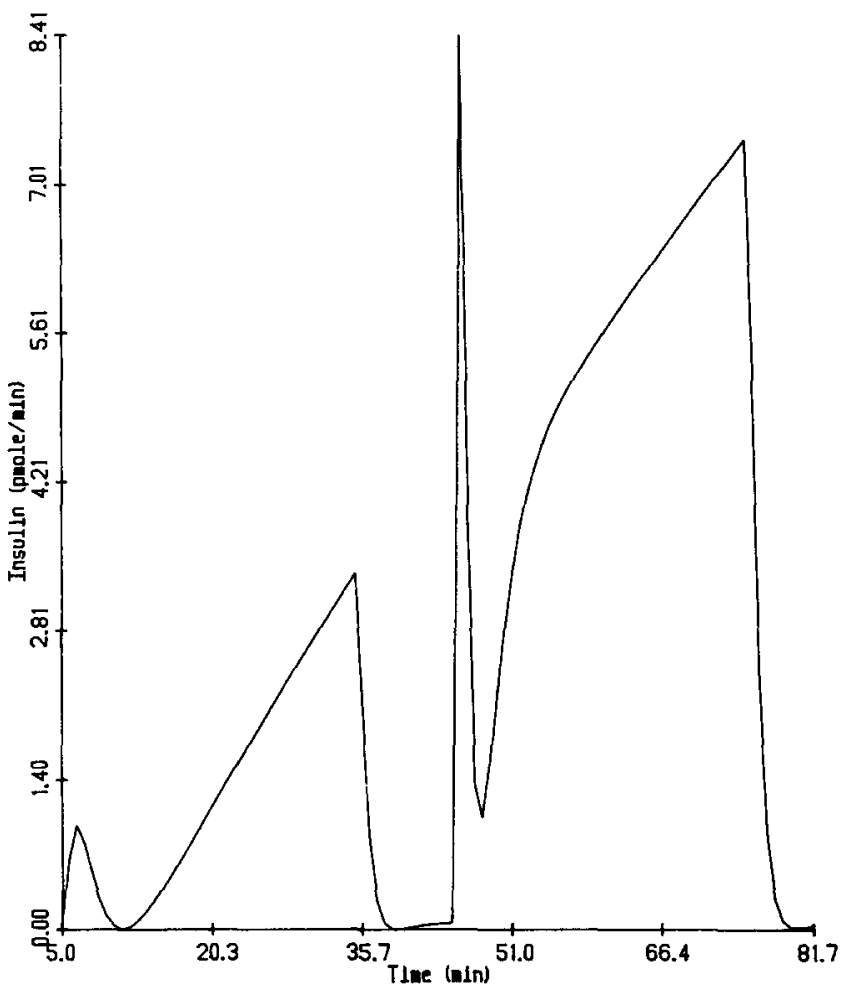

Fig. 4. Computer simulation of glucose-stimulated insulin secretion. Two successive stimuli of glucose were administered (between minutes 5-35 and 45-75) and two biphasic patterns were predicted. The interval between the stimuli was $10 \mathrm{~min}$. The concentration of glucose was changed from $5.6 \mathrm{mM}$ to $16.7 \mathrm{mM}$ during the periods of stimulation. The discontinuity vectors from Table 2 were used.

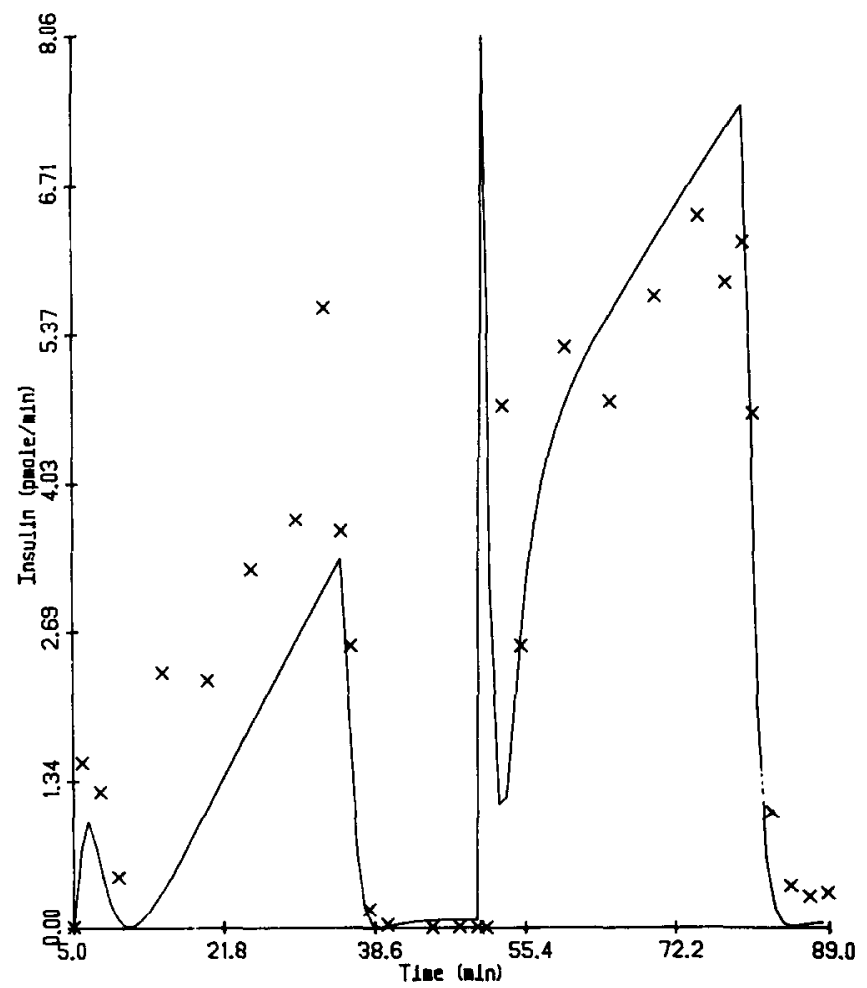

Fig. 5. Raw data $(X)$ and predicted secretory rate (solid line) for glucose-stimulated insulin secretion (experiment P-1092-I). Two successive stimuli of glucose were administered, (between minutes 5-35 and $50-80$ ) and two biphasic patterns were observed. The interval between the stimuli was $15 \mathrm{~min}$. The concentration of glucose was changed from $5.6 \mathrm{mM}$ to $16.7 \mathrm{mM}$ during the periods of stimulation. The discontinuity vectors from Table 2 were used. 


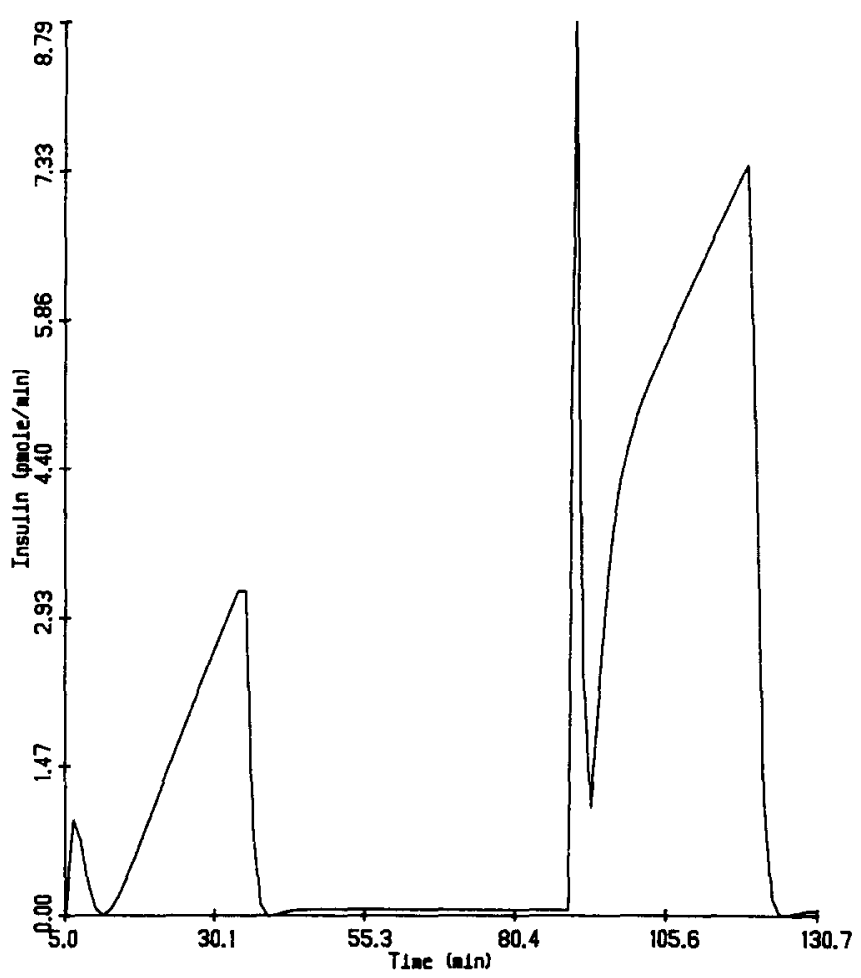

Fig. 6. Computer simulation of glucose-stimulated insulin secretion. Two successive stimuli of glucose were administered (between minutes 5-35 and 90-120) and two biphasic patterns are predicted. The interval between the stimuli is $55 \mathrm{~min}$. The concentration of glucose was changed from $5.6 \mathrm{mM}$ to $16.7 \mathrm{mM}$ during the periods of stimulation. The discontinuity vectors from Table 2 were used.

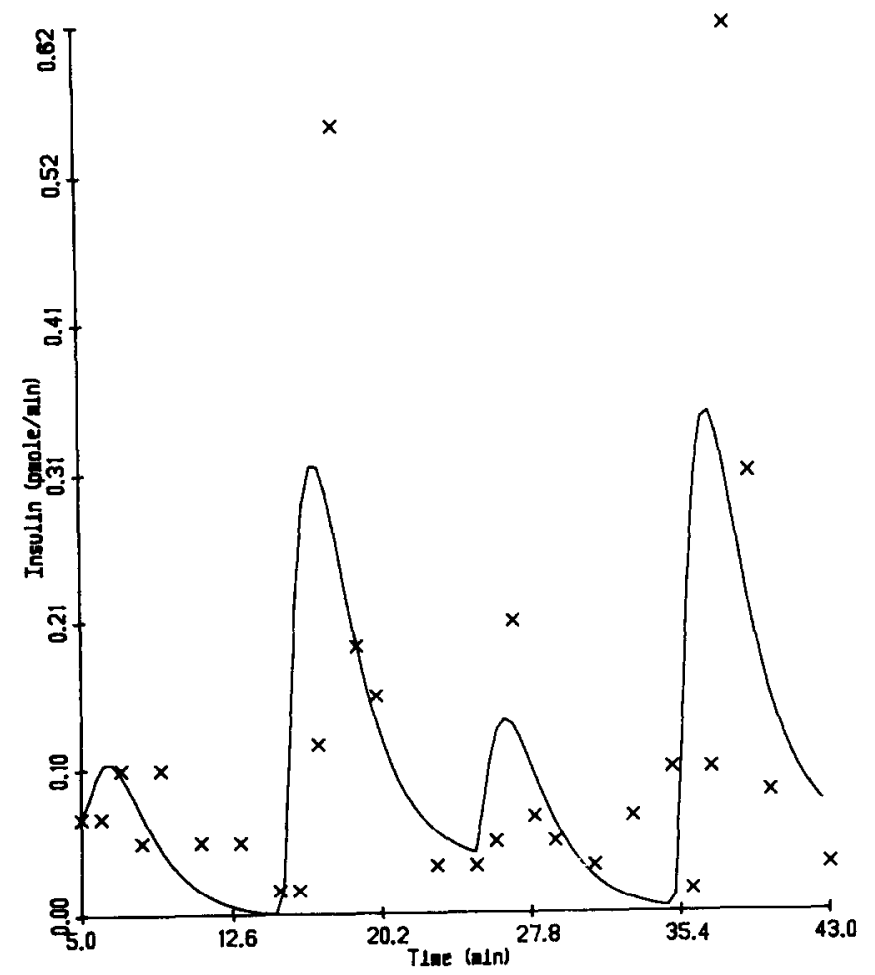

Fig. 7. Raw data $(X)$ and curve approximation (solid line) for leucine-stimulated insulin secretion, in the presence of $5.6 \mathrm{mM}$ glucose (experiment P-701-I). Two successive stimuli of leucine were administered (between minutes 5-15 and 25-35) and two triphasic patterns were observed. The offresponse occurred when the concentration of leucine changed from $5.0 \mathrm{mM}$ to $0.0 \mathrm{mM}$ (minutes $15-25$ and 35-43). The interval between the stimuli was $10 \mathrm{~min}$. 


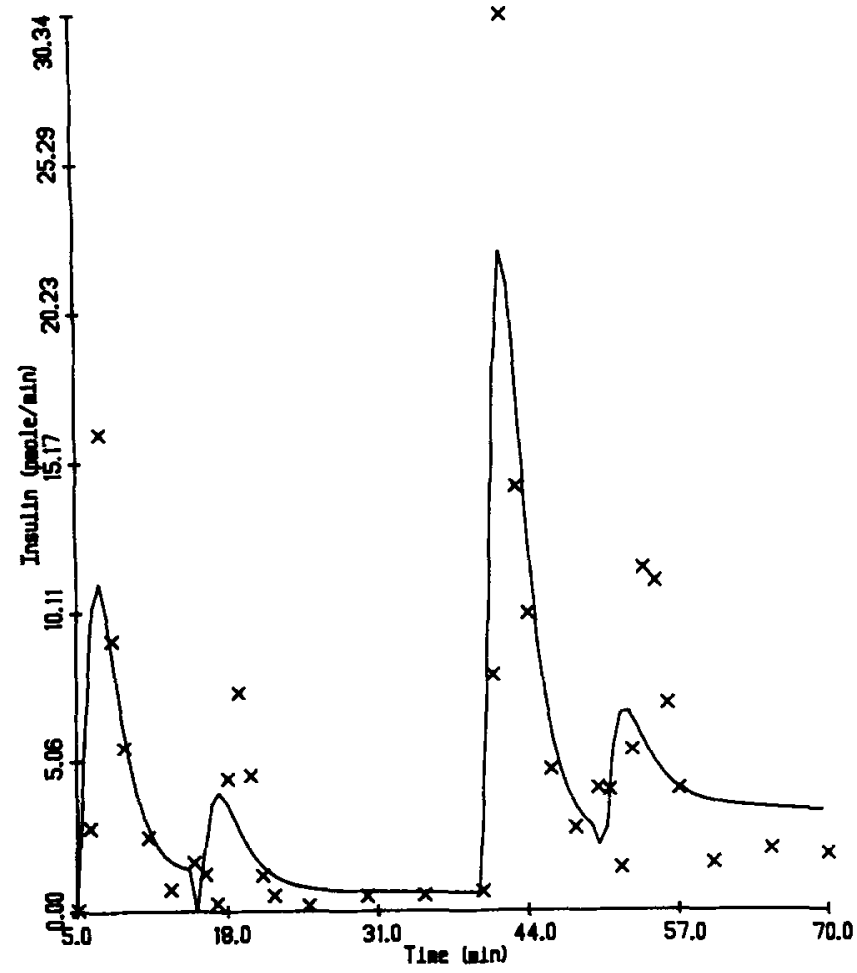

Fig. 8. Raw data $(X)$ and curve approximation (solid line) for leucine-stimulated insulin secretion, in the presence of $5.6 \mathrm{mM}$ glucose (experiment $\mathrm{P}-1379-1$ ). Two successive stimuli of leucine were administered (between minutes 5-15 and 40-50) and two triphasic patterns were observed. The offresponse occurred when the concentration of leucine changed from $20.0 \mathrm{mM}$ to $0.0 \mathrm{mM}$ (minutes 15-25 and 50-60). The interval between the stimuli of leucine was $25 \mathrm{~min}$.

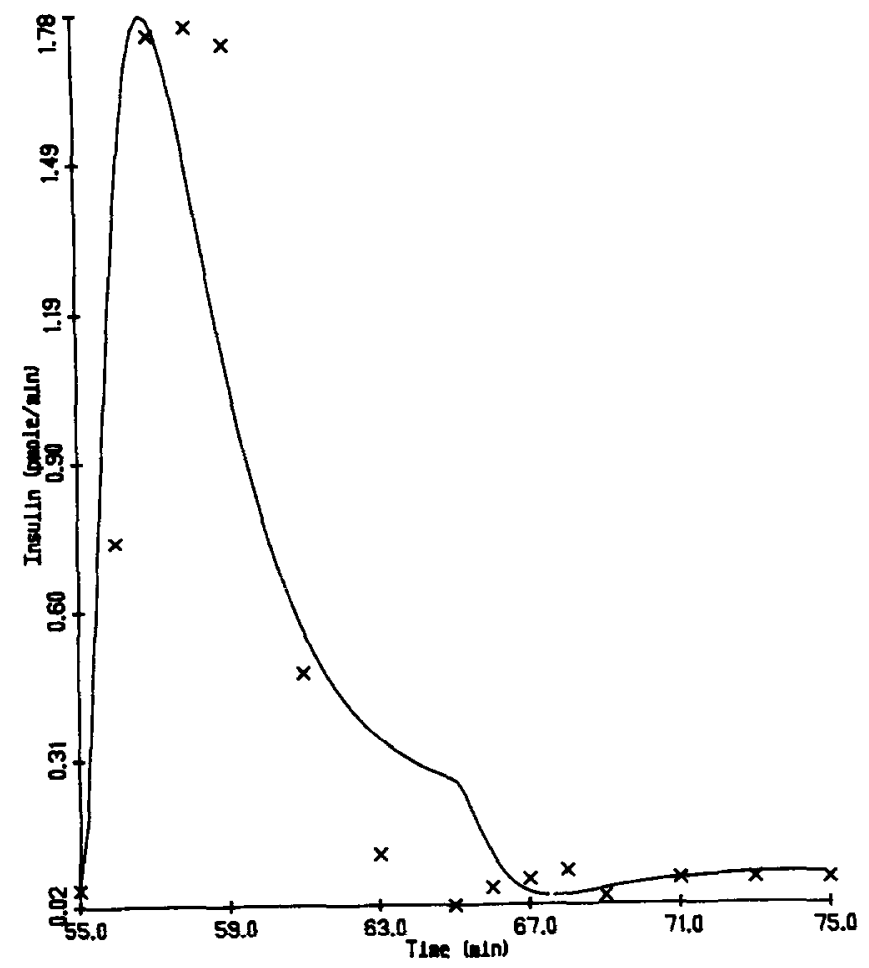

Fig. 9. Raw data $(X)$ and curve approximation (solid line) for leucine-stimulated insulin secretion, in the presence of $10.0 \mathrm{mM}$ theophylline and no glucose (experiment P-767-I). At time $t-55$, the concentration of leucine was raised from $0.0 \mathrm{mM}$ to $5.0 \mathrm{mM}$; at time $t=65$, the concentration of leucine was returned to $0.0 \mathrm{mM}$. 


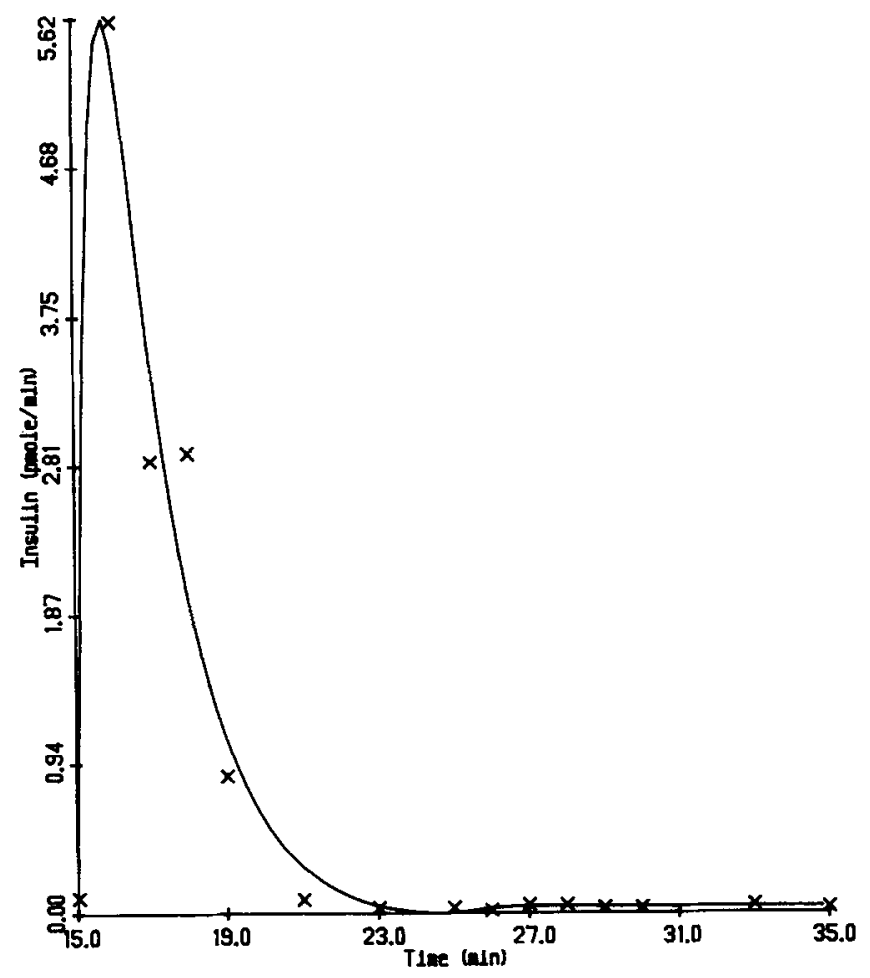

Fig. 10. Raw data $(X)$ and curve approximation (solid line) of a primarily first-phase response. At time $t=15$, the leucine concentration was increased from $0.0 \mathrm{mM}$ to $5.0 \mathrm{mM}$; at time $t=25$, the concentration of leucine was returned to $0.0 \mathrm{mM}$. Theophylline was present in the perfusion medium at a concentration of $10.0 \mathrm{mM}$ and glucose was absent (experiment P-766-I).

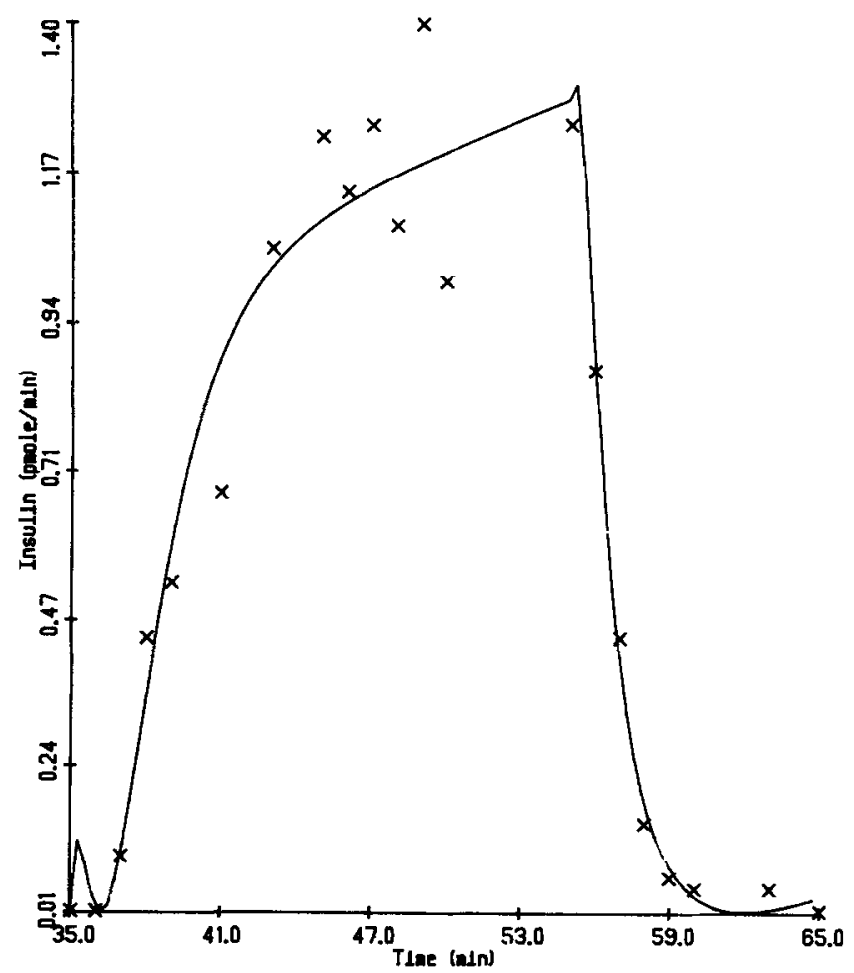

Fig. 11. Raw data $(X)$ and curve approximation (solid line) of a primarily second-phase response. At time $t=35$, the concentration of theophylline was changed $0.0 \mathrm{mM}$ to $10.0 \mathrm{mM}$; at time $t=55$, its concentration was returned to $0.0 \mathrm{mM}$. Glucose was absent from the perfusion medium (experiment P-804-I). 


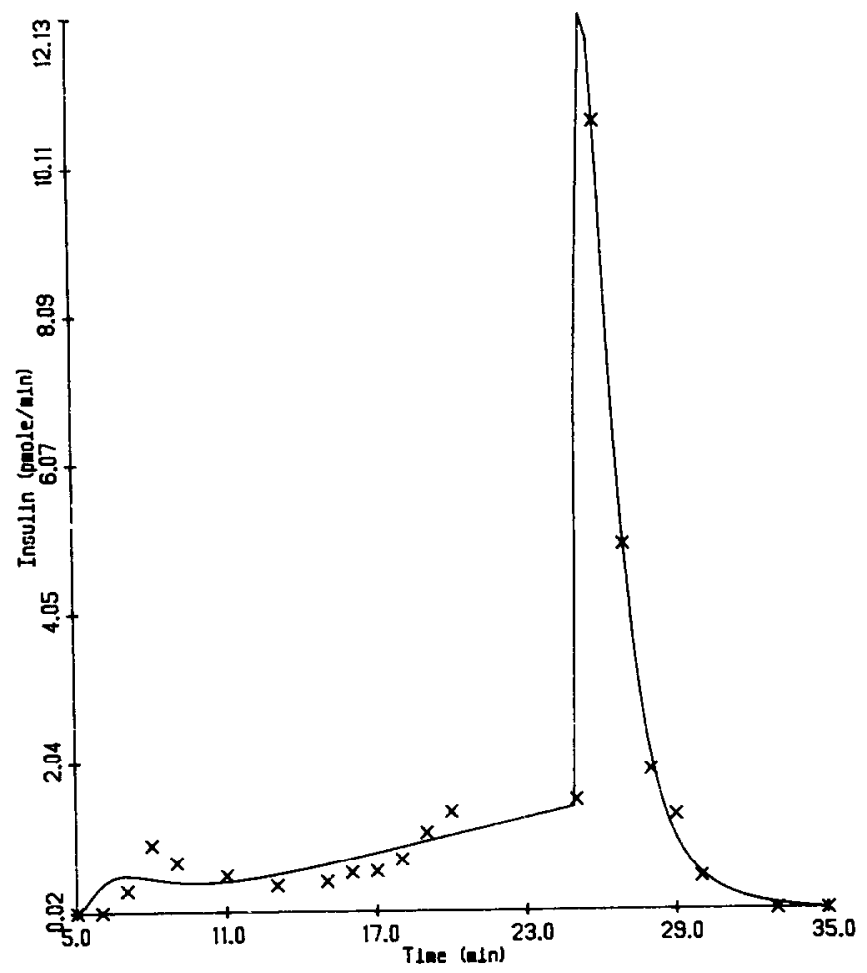

Fig. 12. Raw data $(X)$ and curve approximation (solid line) of a primarily third-phase response (an offresponse). At time $t=5$, the concentration of theophylline was increased from $0.0 \mathrm{mM}$ to $10.0 \mathrm{mM}$; at time $t=25$, its concentration was returned to $0.0 \mathrm{mM}$. Glucose was present throughout the experiment, at a concentration of $5.6 \mathrm{mM}$ (experiment P-747-I).

compared to the experiment in which the concentration of leucine was $5.0 \mathrm{mM}$ (Fig. 7). Glucose was present in the perfusion solution at a concentration of $5.6 \mathrm{mM}$ throughout both experiments.

As presented in Table 4, in the presence of $5.6 \mathrm{mM}$ glucose an increase in the concentration of leucine from $0.0 \mathrm{mM}$ to $20.0 \mathrm{mM}$ (Fig. 8) caused a hundred-fold greater increase in components 1,2 and 3 of the vector $\Delta \mathbf{g}$ than that observed with an increase in the concentration of leucine from $0.0 \mathrm{mM}$ to $5.0 \mathrm{mM}$ (Fig. 7). However, in either case, component 4 of $\Delta \mathbf{g}$ was relatively unaffected.

\section{Uniphasic secretion}

Two types of experiments exhibiting uniphasic responses are presented: one type to study the effects of leucine alone for comparison with its interactions with glucose; and another type to aid in the selection of the matrix $K$ of intercompartmental influence coefficients.

In Fig. 9, an experiment was carried out in the absence of glucose, in which the concentration of leucine was raised from $0.0 \mathrm{mM}$ to $5.0 \mathrm{mM}$ at minute 55 , and then returned to $0.0 \mathrm{mM}$ at minute 65 . The uniphasic secretory response occurred during the administration of leucine; an off-response was not observed following the removal of leucine. In this experiment, theophylline was present in the solutions in order to amplify insulin responses to leucine which are markedly blunted without glucose.

The sign pattern of the first, second, and third components of the vector $\Delta \mathbf{g}$ was +-+ for leucine-stimulated insulin secretion, both in the presence and in the absence of glucose (Figs 7, 8 and 9, Table 4). In the case of glucose-stimulated insulin secretion (Fig. 3), the sign pattern was -+- . 
In the absence of glucose (Fig. 9), an increase in the concentration of leucine from $0.0 \mathrm{mM}$ to $5.0 \mathrm{mM}$ had a (relative) inhibitory effect on compartment 4 . In the presence of $5.6 \mathrm{mM}$ glucose (Fig. 7), a similar increase in the concentration of leucine caused a (relative) stimulation of that compartment.

The second type of experiment was chosen because the patterns of insulin secretion were nearly pure Phase 1, Phase 2 or Phase 3 (Figs 10,11 and 12, respectively). As explained in the Methods section, these patterns were used to correlate perturbations of compartments of the model with phases of insulin secretion.

\section{DISCUSSION}

The proposed model of insulin secretion provides a mathematically-specified mechanism for the biphasic nature of glucose-stimulated insulin secretion. A change in the glucose concentration in the perfusate determines a change in the steady state of the system and an initial displacement of the state of the system, relative to the (new) steady state. The system performs oscillations much as a guitar string will vibrate if it is plucked.* As in the case of a vibrating string, the variable representing the activity level of a compartment can overshoot the equilibrium one or more times before approaching it asymptotically. The late phase of glucose-stimulated insulin secretion corresponds to a gradual approach to the newly established steady state. The acute phase of insulin secretion occurs when the secretory rate overshoots its steady state value twice (once as the rate abruptly increases and once again as it plummets).

The analogy to the vibrating string can be used to explain "pancreatic memory", or the potentiating effect on glucosc-stimulated insulin secretion of prior exposure to the secretagogue. Based on the equations of our mathematical system, the phenomenon of potentiation results from the presence of slower natural frequencies of the secretory system. The origin of the natural frequencies lies in the matrix $K$ of coefficients which relate the influence of one compartment of the system on another, as specified by the equation of state of our system.

In the absence of any information on the biological variables which are represented by the compartments of the present model, a stimulation of a compartment cannot be distinguished from a deinhibition of the same compartment (both of which correspond to a positive-valued component of the vector $\Delta \mathbf{g}$ ). Similarly, a relaxation of a stimulatory influence cannot be distinguished from an increase in an inhibitory influence (both of which correspond to a negative-valued component of the vector $\Delta \mathrm{g}$ ). The aforementioned distinctions could be made, if the identities of the individual compartments were more completely assigned.

We wish to speculate on the identities of the individual or composite metabolic events which may be represented by the compartments of the mathematical model. An increase in the concentration of glucose from $5.6 \mathrm{mM}$ to $16.7 \mathrm{mM}$ stimulates the activity of compartment 4 , whose activity level is supposed to be directly correlated with insulin secretion. A possible mechanism of the direct effect of glucose on insulin release (such as is predicted by the proposed model) may be the activation of the putative "glucoreceptor" on the surface of the insulin-secretory cell (Matschinsky et al. [28], Ashcroft [29]). The glucoreceptor would be sensitive to the concentration of glucose reaching the cell, and would trigger the release of insulin proportionally.

The metabolism of glucose is another important mechanism of glucose-induced insulin release which can be accommodated by the proposed mathematical model (Matschinsky et al. [28]). Since we have tentatively assigned the activation of the glucoreceptor to compartment 4 , we consider which one of the remaining three compartments may represent the influence of glucose metabolism on insulin release. The rate of metabolism of glucose (specifically, of glycolysis) varies directly with the concentration of glucose (Lehninger [30], Vander et al. [31]). Compartments 1 and 3 are inhibited when the concentration of glucose is

\footnotetext{
* A more faithful, though less familiar analogy would be the relaxation oscillations of several masses connected to springs (Tong [26]). In that case, the number of eigenvalues is finite, which is not the case for the guitar string (Bickley and Talbot [27]).
} 
increased from $5.6 \mathrm{mM}$ to $16.7 \mathrm{mM}$, and stimulated when the concentration of glucose is decreased from $16.7 \mathrm{mM}$ to $5.6 \mathrm{mM}$. Compartment 2, on the other hand, is stimulated when the concentration of glucose is increased and inhibited when the concentration of glucose is decreased. Therefore, compartment 2 is the most reasonable choice for a compartment to represent the net rate of glucose catabolism.

The interpretations of compartments 2 and 4 which we propose are in agreement with the suggestion (Tai et al. [6]) that when the concentration of leucine is decreased in the presence of $5.6 \mathrm{mM}$ glucose, glucose metabolism increases, thereby causing an off-response in insulin secretion. Compartment 2 of our model, to which we have tentatively assigned the metabolism of glucose, is inhibited by increases in the concentration of leucine, both in the presence and in the absence of glucose (Table 4). When the concentration of leucine is decreased, compartment 2 is stimulated (because the sign of each component of the vector $\Delta \mathbf{g}$ is reversed). Therefore, the off-response occurs when the decrease in the concentration of leucine causes a (relative) stimulation of compartment 2 .

The predictions of the model with respect to the change of the potentiation of insulin secretion over time are in substantial agreement with experimental findings. Experiments performed by Grill et al. [4] with the perfused pancreas of fasted rats show that the insulinsecretory response to the second dose of glucose is $198 \%$ of the response to the first dose when the interval between the doses is $10 \mathrm{~min}$, and $181 \%$ when the interval is $60 \mathrm{~min}$. Our model predicts no change in the relative potentiated response when the time interval is increased from $10 \mathrm{~min}$ to $55 \mathrm{~min}$.

The proposed theoretical model expands the usefulness of mathematical modeling in the study of the mechanisms of insulin secretion. The application of the model to glucosestimulated insulin secretion was a necessary test of the validity of the theory underlying the model. Other models of glucose-stimulated insulin secretion (Grodsky [8], Cerasi [11]) are sufficient in this respect. The variety of secretory patterns which have been observed (Matschinsky et al. [3]) requires a model capable of exhibiting these patterns and an algorithm for adapting the model to each type of pattern. We have developed a model and an algorithmic technique to meet these needs, in order to probe more deeply into the mechanisms underlying hormone secretion.

\section{SUMMAR Y}

We have presented a new mathematical model of insulin secretion from the pancreatic islets of Langerhans. The model has four compartments; each compartment represents an important, hypothetical biological energy transformation which affects the secretory rate. The activity of compartment 4 is correlated with the insulin-secretory rate. The proposed model reproduces experimentally observed patterns of insulin secretion, such as the acute and late phases of release in response to glucose or to leucine; the "off-response" that occurs upon the removal of certain stimuli, such as leucine; and the long-term potentiating effect of repetitive administration of secretagogues.

In the context of the proposed model, in the absence of glucose an increase in the concentration of L-leucine stimulates, inhibits, stimulates, and inhibits compartments 1, 2, 3 and 4, respectively. In the presence of glucose the effects of leucine are modified so that compartment 4 is stimulated rather than inhibited. Thus, the off-response to leucine, which occurs in the presence but not in the absence of glucose, is associated with a change in the actions of leucine on a single compartment. Raising the concentration of glucose causes the inhibition, stimulation, inhibition, and stimulation of compartments $1,2,3$ and 4 , respectively. Compartment 4 of the model may represent the balance of stimulatory and inhibitory forces within the pancreatic islets.

Acknowledgements--The work presented in this report was carried out in partial fulfillment of the Degree of Doctor of Philosophy at the University of Michigan (D. Cohen). The Doctoral Dissertation was entitled "A Steady-StateTransition Model of Insulin Secretion", Department of Computer and Communication Sciences, The University of Michigan, Ann Arbor, Michigan, 1981 (printed as the Logic of Computers Group Technical Report No. 239).

The experimental portion of the study was supported in part by USPHS grants AM-02244 and AM-21192 from 
the National Institute of Arthritis, Diabetes, Kidney, and Digestive Diseases. Dr. Cohen was supported in part by the National Science Foundation Grants MCS76-04297 and MCS78-26016.

Dr. Cohen thanks the following people for their help during his Ph.D. work: John H. Holland, Ph.D., Sumer

Belbez Pek, M.D., Arthur W. Burks, Ph.D.. William E. M. Lands, Ph.D., and Aaron I. Vinik, M.D.

Dr. Cohen and Dr. Pek thank John H. Holland, Ph.D. for reading the manuscript prior to its submission.

\section{REFERENCES}

1. C. J. Hedeskov, Mechanism of glucose-induced insulin secretion, Physiol. Rev. 60, 442 (1980).

2. P. H. Smith and D. Porte, Jr., Neuropharmacology of the pancreatic islets, Ann. Rev. Pharmac. Toxicol. 16, 269 (1976).

3. F. M. Matschinsky, R. Fertel, J. Kotler-Brajtburg, S. Stillings, J. Ellerman, F. Raybaud and J. HolowachThurston. Factors governing the action of small calorigenic molecules on the islets of Langerhans, Proceedings of the Eighth Midwest Conference on the Thyroid and Endocrinology (October 9-10,1972, University of MissouriColumbia). pp. 63-87. X. J. Musacchia and R. P. Breitenbach, Eds., University of Missouri-Columbia (1973).

4. V. Grill, U. Adamson and E. Cerasi, Immediate and time-dependent effects of glucose on insulin release from rat pancreatic tissue, $J$. clin. Intest. 61, 1034 (1978).

5. D. J. Porte, Jr., P. H. Snith and J. W. Ensinck, Neurohumoral regulation of the pancreatic islet A and B cells, Metabolism 25 (Suppl. 1), 1453 (1976).

6. T.-Y. Tai, S. Pek, and J. C. Santiago, L-leucine-induced secretion of glucagon and insulin, and the "off-response" to L-leucine in vitro. II. The role of D-glucose, Endocrinology 103, 1219 (1978).

7. G. Grodsky, Insulin and the pancreas, Vitams Horm. 28, 37 (1970).

8. G. Grodsky, A threshold distribution hypothesis for packet storage of insulin and its mathematical modeling, $J$. clin. Invest. 51, 2047 (1972)

9. R. N. Bergman and J. Urquhart, The pilot gland approach to the study of insulin secretory dynamics, Recent Prog. Horm. Res. 27, 583 (1971).

10. E. Cerasi, G. Fick and M. Rudemo, A mathematical model for the glucose induced insulin release in man, Eur. $J$. clin. Invest. 4, 267 (1974).

11. E. Cerasi, Insulin secretion: mechanism of the stimulation by glucose, Q. Rev. Biophys. 8, 1 (1975).

12. V. Licko and A. Silvers, Open-loop glucose-insulin control with threshold secretory mechanism: Analysis of intravenous glucose tolerance tests in man, Mathl Biosci. 27, 319 (1975).

13. J. R. Guyton, R. O. Foster, J. S. Soeldner, M. H. Tan, C. B. Kahn, L. Koncz and R. E. Gleason, A model of glucose-insulin homeostasis in man that incorporates the heterogeneous fast pool theory of pancreatic insulin release, Diabetes 27, 1027 (1978).

14. P. Hagander, K.-G. Tranberg, J. Thorell and J. Distefano III, Models for the insulin response to intravenous glucose, Mathl Biosci. 42, 15 (1978).

15. C. Cobelli, G. Pacini and A. Salvan, On a simple model of insulin secretion, Med. Biol. Engny Comput. 18, 457 (1980).

16. G. Toffolo, R. N. Bergman, D. T. Finegood, C. R. Bowden and C. Cobelli, Quantitative estimation of beta cell sensitivity to glucose in the intact organism: A minimal model of insulin kinetics in the dog, Diabetes 29, 979 $(1980)$.

17. R. N. Bergman and R. J. Bucolo. Nonlinear metabolic dynamics of the pancreas and liver, J. Dyn. Syst., Measmt Cont. 95, $296(1973)$.

18. S. Pek, J. C. Santiago and T.-Y. Tai, L-leucine-induced secretion of glucagon and insulin and the "off-response" to L-leucine in vitro. I. Characterization of the dynamics of secretion, Endocrinology 103, 1208 (1978).

19. M. M. C. I andgraf-I eurs, R. Landgraf, R. Daffner and R. Hörl, The stimulatory and inhibitory function of theophylline on amino acid-induced insulin release: Studies with the perfused rat pancreas, Endocrinology 97 , 1416 (1975).

20. D. Cohen, A Steady-state-transition model of insulin secretion, Ph.D. Dissertation, Department of Computer and Communication Sciences, The University of Michigan, Ann Arbor, MI (1981).

21. C. Lanczos, Applied Analysis. p. 272. Prentice Hall, Englewood Cliffs, NJ (1964).

22. R. Hamming. Numerical Methods for Scientists and Engineers. 2nd ed., p. 623. McGraw-Hill, New York (1973).

23. A. Ravindran, A computer routine for quadratic and linear programming problems. Algorithm 431, Collected Algorithms from CACM, Vol. 2, pp. 431-P1-0, 431-P2-0, 431-P3-R2. ACM, New York (1978).

24. A. Ravindran, Remark on Algorithm 431, Collected Algorithms from CACM, Vol. 2, p. 431-P3-R2. ACM, New York (1978).

25. L. G. Proll, Remark on Algorithm 431, Collected Algorithms from CACM, Vol. 2, pp. 431-P3-R2, 431-P4-0. ACM, New York (1978).

26. K. N. Tong, Theory of Mechanical Vibration. John Wiley, New York (1960).

27. W. G. Bickley and A. Talbot, An Introduction to the Theory of Vibrating Systems. Clarendon Press, Oxford (1961).

28. F. M. Matschinsky, R. Landgraf, J. Ellerman and J. Kotler-Brajtburg, Glucoreceptor mechanisms in the islets of Langerhans, Diabetes 21 (Suppl. 2), 555 (1972).

29. S. J. H. Ashcroft, Glucoreceptor mechanisms and the control of insulin release and biosynthesis, Diabetologia 18, 5 (1980).

30. A. L. Lehninger, Biochemistry: The Molecular Basis of Cell Structure and Function. 2nd ed., p. 423. Worth, New York (1975).

31. A. J. Vander, J. H. Sherman and D. S. Luciano, Human Physiology: The Mechanisms of Body Function. 2nd ed., p. 83. McGraw-Hill, New York (1975). 
About the Author-David Marshall Cohen received the B.A. degree in Chemistry from Case Western Reserve University in 1974, and the M.S. and Ph.D. degrees in Computer and Communication Sciences from The University of Michigan in 1978 and 1981, respectively.

Dr. Cohen spent the next two years as an Assistant Professor of Computer Science at the University of Michigan-Flint. He is presently employed as a Senior Software Engineer with Applied Dynamics International. His current research interests include biological modeling and simulation, compiler construction, artificial intelligence, and foundations of computer science

Dr. Cohen is a member of the American Association for the Advancement of Science, the American Association for Artificial Intelligence, the Association for Computing Machinery, the Institute of Electrical and Electronics Engineers Computer Society and Phi Beta Kappa.

About the Author-Sumer Belbez Pek received the degree of Doctor of Medicine from the University of Munich, Germany in 1959. He completed specialty training in Internal Medicine followed by an Endocrinology and Metabolism Fellowship at the University of Michigan. In 1969 he joined the Faculty of the University of Michigan Medical School as Assistant Professor of Internal Medicine. In 1973 and 1979 respectively, he was promoted to Associate Professor and full Professor of Internal Medicine at this Institution.

Dr. Pek's research activities focus on the mechanisms of secretion of pancreatic islet hormones. His early work was on the regulation of secretion of insulin and glucagon by amino acids and hormones. Subsequently, he provided evidence that glucagon was also produced in and released from extrapancreatic sites. More recently, he has been investigating the role of arachidonic acid metabolism, particularly of prostaglandins and leukotrienes in regulation of the function of the endocrine pancreas. 